\title{
HORIZONS
}

\section{TRIALS AND TRIBULATIONS}

The medical maxim of 'First do no harm' is never more relevant than during development of a medicinal agent or intervention, whether it be drug, vaccine, diagnostic or procedure. Over many years a phased clinical trial process has been developed along with safety and monitoring processes to ensure human subject dignity, privacy and autonomy, and the minimisation of risk as well as research integrity.

Before any testing in human subjects the process involves pre-clinical work, including toxicity studies, wherever possible in animal models. Human subject testing then follows with phase 1 trials, often in small numbers $(10-100)$ of adult volunteers who may or may not have the condition under consideration, and under very carefully monitored trial conditions. This phase tests safety only. Phase 2 is conducted in slightly larger groups (100s) of individuals (now possibly involving the target population), again mostly testing safety, but also ideal dose and possibly some indicators of activity. In Phase 3, the well-known efficacy studies, much larger groups (100s - 1000s) of the target population are included and while safety should still be carefully monitored the main question is efficacy. Efficacy can be tested most rigorously if a comparison group is included, usually receiving the 'standard of care' in order to allow the trial to be performed adequately and without unfair disadvantage to the experimental group.

Every phase of the process requires careful review by independent regulatory and ethical groups to ensure that all considerations of safety and indicators to move forward are met. In dose escalation studies, safety pauses should again be incorporated. Where possible independent safety monitoring groups should have access to trial data to ensure that safety is reviewed objectively and studies modified or stopped for reasons of safety and/or efficacy.

This process is incorporated within the concept of 'Good Clinical Practice', and I would contend that somehow GCP has got lost in the minutiae of whether our case report forms are filled out perfectly in black or blue ink. While detail is important and an important element of clinical research, the primary purpose of GCP is to meet the highest levels of human subject protection while conducting well-designed clinical trials that inform the developmental process.

Two events in the last week have highlighted these issues. First is the recent disaster at Northwick Park Hospital, London. Six healthy young men - including British Asians, an Australian, a New Zealander, and a South African, contracted by the US drug testing company Parexel to test the anticancer drug TGN1412 - went into sudden multiple organ failure as a result of unexpected massive inflammatory reactions. Why did the rigorous rules laid down by the Medicines and Healthcare Products Regulatory Agency (MHRA) fail to halt these dreadful events? The MHRA has begun an investigation into the calamity, which should provide answers to these questions. It may be months before any inquiries give answers, and in the meantime these previously healthy volunteers are fighting for their lives.
There are three reported questionable aspects to this disaster:

1. The drug is a monoclonal antibody that targets the CD28 site on the surfaces of T cells, kick-starting the body's immune system into action. One could argue for a trial design for early phase 1 studies to be conducted not in healthy volunteers whose immune systems are normal and vigorous but in cancer patients or individuals with autoimmune disorders. Because the monoclonal is humanised it is not always easy to interpret animal toxicity data. Finally, new technology involving microdosing where local systems are set up in blisters on the skin may give more information in trials of this kind before proceeding to normal dosing.

2. Secondly, the dose escalation in this study was reportedly performed inordinately rapidly and, some would argue, without sufficient safety pause.

3. Finally, the study participant recompensation for the trial was apparently in the order of $£ 2000$ each, and it has been argued that inducements of this magnitude may cloud volunteers' ability to judge personal risk and lead to non-disclosure of pre-existing medical conditions, etc.

The next item of news is local: weekend papers ran a story on senior members of government who have supported use of unproven traditional medicines for AIDS, and this at the expense of clinically tried and tested licensed antiretroviral agents.

Ubhejane is the secret recipe of Zeblon Gwala, a former truck driver from KZN who claims that its ingredients came to him in a dream from his healer grandfather. Apparently he personally collects the 89 herbal ingredients from all over South Africa and mixes them manually. It is then sold in unlabelled 2-litre plastic bottles for R342 each and lasts 2 weeks. More seriously, aside from any possible toxicity, patients commencing use of Ubhejane are reportedly encouraged to stop or not use prescribed antiretrovirals.

The Sunday Times reported that KZN Minister of Health, the eThekwini Mayor and the special advisor to the KZN Premier have openly encouraged people to take this product despite no evidence of conventional clinical testing for either safety or efficacy. If this is true it is both dangerous and unethical, particularly if it is recommended as a substitute for a proven life-saving treatment.

Mr Gwala claims that he has records of all patients on this treatment, but could not supply details to the Sunday Times. Gwala is quoted as saying: 'I do keep files but there are so many and I don't know which has these things in it'.

With 5 - 6 million South Africans infected with HIV we would be ecstatic if Ubhejane or some other indigenous medicine could be found to be part of the armamentarium against HIV, but in order to prove any new product's worth and safety, and before wild claims of efficacy are made to a desperate public, we need to follow drug testing protocols developed to protect the public.

LINDA-GAIL BEKKER

Managing Editor

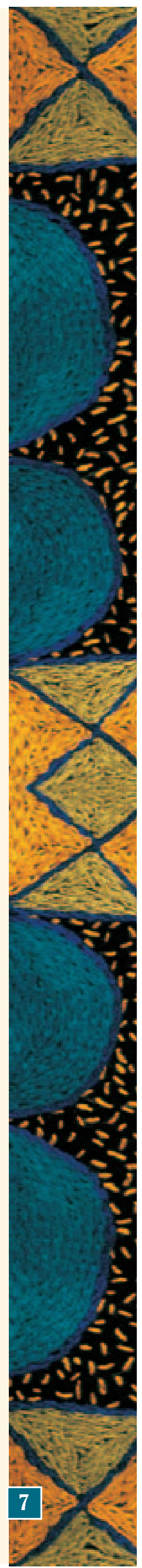

